\title{
Effects of a 12-Week Systematized Yoga Intervention on Health-Related Physical Fitness in Healthy Adults
}

\author{
Juliana Costa Shiraishi*, André Bonadias Gadelha, Lídia Mara Aguiar Bezerra, \\ Luiz Guilherme Grossi Porto
}

Faculty of Physical Education, University of Brasília, Brasília, Brazil

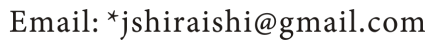

How to cite this paper: Shiraishi, J. C., Gadelha, A. B., Bezerra, L. M. A., \& Porto, L. G. G. (2017). Effects of a 12-Week Systematized Yoga Intervention on Health-Related Physical Fitness in Healthy Adults. $A d$ vances in Physical Education, 7, 27-37. https://doi.org/10.4236/ape.2017.71003

Received: December 7, 2016

Accepted: February 12, 2017

Published: February 15, 2017

Copyright (c) 2017 by authors and Scientific Research Publishing Inc. This work is licensed under the Creative Commons Attribution International License (CC BY 4.0).

http://creativecommons.org/licenses/by/4.0/

\begin{abstract}
Regular physical activity has been shown to improve overall physical fitness. The long-term adherence to physical activity is a big challenge for health maintenance; so, pleasure activities, as Hatha Yoga (yoga), may represent a good alternative on both individual and public contexts. Yoga is an ancient activity designated to both health and unhealthy individuals. It integrates physical, mental, and spiritual components and may improve aspects of health. The aim of the present study was to evaluate the effects of a 12-week systematized yoga intervention on health-related physical fitness components assessed by body mass, body mass index (BMI), waist circumference (WC), relative body fat, abdominal endurance, upper body endurance, hamstring flexibility, and cardiorespiratory fitness. The study was performed at University of Brasília, Faculty of Physical Education, Distrito Federal, Brazil. Twenty-five young healthy yoga novices ( $22.36 \pm 2.40$ years), both gender, volunteered to participate in this study. The intervention was based on 50 minutes yoga class, twice a week for 12 weeks, involving physical poses, meditation, and relaxation. Measurements were performed one week prior to and one week after the yoga intervention. Collecting data included age, gender, height, body mass, body fat estimates and physical fitness tests. Body fat percentage was determinated by the measure of skinfolds at seven sites. Muscle strength/endurance was evaluated by push-up and sit-up tests. Hamstring flexibility was assessed using sit-and-reach test protocol. The 12-minutes Cooper test was performed to estimate cardiorespiratory fitness. Participants that attended at least $75 \%$ of the total sessions were included in the study results. The results showed 1.3 (1.0 4.0) $\mathrm{cm}$ decrease in WC and $0.7(0.9-1.5)$ decrease in body fat percentage, and $7.8(2.0-5.0) \mathrm{cm}$ increase in hamstring flexibility and $3.0(1.0-4.0)$ in abdominal endurance, after the yoga intervention $(\mathrm{p}<0.01)$. In conclusion, the present study found that a 12 -week yoga program improved physical fit-
\end{abstract}


ness in young healthy subjects.

\section{Keywords}

Yoga, Physical Activity, Fitness, Flexibility

\section{Introduction}

There is a broad consensus demonstrating that regular physical activity (PA) can lead to a range of health benefits in adults (ACSM, 2011) and even for elderly people (Rooney, 1993). Moreover, there is positive association between increased levels of PA, exercise participation and improved health in older adults (Taylor, 2014). More recently, low PA has been recognized as an important issue even among children. Apart from that, low levels of PA at 12 years old might predict future unfavorable health behavior in the adult age (Gadin \& Hammarström, 2002). In that scenario, physical activity promotion is one of the most difficult and important challenge in lifestyle change (Erikssen, 2001). Despite some good PA promotion programs around the world, as the Agita São Paulo (Matsudo, Matsudo, Araújo et al., 2010) and Bogotá Bicycle Paths Initiatives (Torres, Sarmiento, Stauber, \& Zarama, 2013), physical inactivity is still a major public health problem. To face that complex human behavior, different strategies to promote PA must be evaluated (Hallal, Andersen, Bull et al., 2012; Lee, Shiroma, Lobelo et al., 2012). There are several pathways of regular PA broadly performed that deserve scientific evaluations. Usually, many people seek alternative paths of physical exercise as Hatha Yoga classes. Considering that long-term adherence to PA is beneficial for the maintenance of health benefits (Allen \& Morey, 2010), and might be the biggest challenge for reducing physical inactivity prevalence, activities that are associated with pleasure and well being, as Hatha Yoga, may represent good alternatives on both individual and public health contexts.

The popularity of yoga is evident with estimation that in 2012 there were about 10.4 million adults' practitioners in the United States, corresponding to $5.1 \%$ of the US population (Birdee, Legedza, Saper et al., 2008). In England, the proportion of yoga practitioners was $1.11 \%$ in 2008 (Ding \& Stamatakis, 2014). Yoga, as a mind-body therapy, may have better adherence than other physical interventions, because of the emphasis on the process instead of on the outcome (Flegal, Kishiyama, Zajdel et al., 2007). Further, Hatha Yoga is also a nonpharmacological intervention recommended to both health and unhealthy individuals. It integrates physical, mental, and spiritual components which improves different aspects of health. Yoga has been associated with enhancement of muscular strength, body composition, cardiorespiratory fitness, sleep pattern and quality of life besides a reduction in stress, anxiety, depression and chronic pain (Woodyard, 2011). In addition, yoga has been pointed as a positive contribution for individuals with overweight, obesity, acute and chronic pain, hypertension, diabetes mellitus, and dyslipidemia (Yang, 2007; Aljasir, Bryson, \& Al-Shehri, 
2008; Posadzki, Ernst, Terry, \& Lee, 2011; Seo, Lee, Figueroa et al., 2012; McCall, Ward, Roberts, \& Heneghan, 2013).

Beyond its physical benefits, yoga is a PA that is accessible to almost everyone, and practitioners tend to maintain a regular practice as a consequence of the feeling of well-being (Kristal, Littman, Benitez, \& White, 2005). A typical yoga class contemplates a combination of physical postures, breathing techniques, and meditation (Bankar, Chaudhari, \& Chaudhari, 2013). In 2011, Field suggested that yoga might improve stretching and muscle toning, keeping the spine and joints flexible (Field, 2011). Additionally, it is suggested that yoga practice may contribute to long-term weight maintenance, by improving exercise capacity and expending sufficient energy (calories) to contribute to weight control (Kristal, Littman, Benitez, \& White, 2005). Therefore, it may be effective in promoting weight loss and/or improvement in body composition (Rioux \& Ritenbaugh, 2013).

Due to the limited number of studies focused on physical outcomes associated with yoga, the available scientific evidence is insufficient to state formal recommendation on the role of yoga in promotion health-related fitness and health. Apart from that, some frequent methodological issue may contribute to that condition, as the short intervention period commonly used (6 to 10 weeks) (Buffart, Uffelen, Riphagen et al., 2012). Given the importance of both PA as well its adherence, studies with Hatha Yoga are timely and scientifically important since it would be an attractive tool against the aforementioned unhealthy lifestyle. It could benefit both healthy and unhealthy adults. Thus, the aim of the present study was evaluate the effects of a 12 -week systematized yoga intervention on health-related physical fitness. It was hypothesized that the systematized intervention of Hatha Yoga is sufficient to improve health-related physical fitness components.

\section{Methods}

\subsection{Participants}

A total of 25 subjects $(22.36 \pm 2.40$ years; $1.66 \pm 0.10 \mathrm{~m})$ volunteered to participate in this study. Thus, the study subjects comprise a non-probabilistic and convenience sample. Participants were all young yoga novices, both gender (17 females and 5 males), and college students of University of Brasília. The Ethics Committee of the University of Brasília approved the protocol for this study, and it was performed according to the Helsinki Declaration of 1975. Participants were explained about the purpose and procedures of the study, and provided written informed consent to participate.

\subsection{Measures}

Measurements were performed during one week prior to and one week after the 12-week yoga intervention. The tests were done under standard laboratory conditions after familiarizing the subjects with the testing procedures. Collecting data included age, gender, height, body mass, and health-related physical fitness, 
which comprises 5 components: body composition, muscle strength, muscle endurance, flexibility, and cardiorespiratory fitness (CRF) (Caspersen, Powell, \& Christenson, 1985). The body mass index (BMI) was calculated using the standardized formula:

$$
\mathrm{BMI}=\operatorname{body} \operatorname{mass}(\mathrm{kg}) / \text { height }^{2}(\mathrm{~m}) \text {. }
$$

WC was measured at the level midpoint between the lower rib margin and the iliac crest with participants in standing position. Body fat percentage was determinated by the measure of skinfolds at seven sites: triceps, subscapular, chest, thoracic, suprailiac, abdomen, and thigh. Muscle strength/endurance was evaluated by push-up (upper body endurance) and sit-up (abdominal endurance) tests, during 1 minute, with the maximum number of repetitions being recorded. Hamstring flexibility was assessed using sit-and-reach test protocol (Hoeger \& Hoeger, 2010), with two trials and keeping the best one. The 12-minutes Cooper test (Cooper, 1968) was performed in the 400 meters athletics track to estimate cardiorespiratory fitness. A brief warm-up was allowed to all participants and the maximum distance covered by running and/or walking at the end of the 12 minutes period was recorded. Thus, $\mathrm{VO}_{2 \text { peak }}$ was estimated by the following equation, proposed by Cooper (1968):

$$
\mathrm{VO}_{2 \text { peak }}=[\text { distance }(\text { meters })-504] / 45 \text {. }
$$

\subsection{Procedures}

Participants started their 12-week yoga intervention on the following week after a pre-intervention testing session. The intervention sessions took place at University of Brasília, Faculty of Physical Education. Participants that attended at least $75 \%$ of the total sessions over the course of 12 weeks were included in the study results. The intervention was based on 50 minutes Hatha Yoga class. A certified Hatha Yoga instructor led it for 3 months twice per week. The classes were conducted on a standardized sequence: sited meditation (5 minutes), physical poses (40 minutes) and lay down relaxation (5 minutes). The sequentially physical poses performed were: sun salutation, mountain, warrior, tree, triangle, crow, snake, pigeon, frog, seated forward bend, camel, half twist, butterfly, shoulder stand, fish, and corpse. Post-intervention testing took place after one week of the completion of the 12-week intervention, and followed the same protocol as for the pre-intervention testing session.

\subsection{Statistical Analyses}

Data were expressed as median (minimum and maximum values). The sample normality was tested using the Shapiro-Wilk test. Thus, the difference between pre and post intervention was performed by paired Wilcoxon test to all variables. Of note, BMI, WC, body fat percentage, and abdominal endurance showed normal distribution, hence they were primarily analyzed as parametric variables; however there was no difference between parametric and nonparame- 
tric tests considering the aforementioned variables. Cohen's $d$ was calculated to identify the effect size of training program after 12-week yoga intervention. The significance level was set at $p<0.05$ and all analyses were conducted using the Statistical Package for Social Sciences version 20.0 (SPSS Inc., Chicago, IL, USA).

\section{Results}

All subjects fulfilled the minimum preset attendance. Nevertheless, there were sample losses of about 3 subjects because they lacked the post measurement meeting. Noteworthy, these subjects were contacted and all absences were explained by personal reasons. Moreover, the sample was also composed by both male and female genders, 5 and 17, respectively. Although all statistical analysis were also performed separately by genders, all data are presented only for the whole group (male and female) since there were no differences between genders $(p>0.05)$.

Table 1 shows dependent variables at pre and post 12-week yoga program. WC and body fat percentage were significantly decreased after the 12-week yoga program. Moreover, hamstring flexibility and abdominal endurance were statistically increased. Even though the other variables did not show statistical difference after the intervention program, it was observed small effect size in both upper body endurance and BMI after the yoga program. Nevertheless, there were no other statistical differences.

\section{Discussion}

The hypothesis of the present study was that the systematized intervention of Hatha Yoga would be able to promote positive changes in health-related physical fitness. According to our results, a statistically significant reduction on WC and body fat percentage, and an increase on hamstring flexibility and abdominal endurance were observed after experimental protocol. These findings are consistent

Table 1. Descriptive and dependent variables pre and post a 12-week yoga program. Data were expressed as median (minimum and maximum values). The effect size of intervention was expressed to dependent variables analyzed.

\begin{tabular}{cccc}
\hline Variable & Pre Yoga Program & Post Yoga Program & ES \\
\hline$n$ & 25 & 22 & - \\
Body mass $(\mathrm{kg})$ & $58.0(46.8-90.3)$ & $60.0(45.6-90.7)$ & 0.02 \\
BMI $\left(\mathrm{kg} / \mathrm{h}^{2}\right)$ & $21.9(18.0-31.5)$ & $21.6(17.9-32.3)$ & 0.14 \\
WC $(\mathrm{cm})$ & $67.1(59.0-92.0)$ & $65.8(58.0-96.0)^{*}$ & 0.45 \\
Body Fat $(\%)$ & $22.3(16.9-36.6)$ & $21.6(16.0-35.1)^{*}$ & 0.54 \\
Hamstring flexibility $(\mathrm{cm})$ & $27.0(14.0-42.5)$ & $34.8(16.0-47.5)^{*}$ & 0.81 \\
AE (repetitions) & $24.0(15.0-52.0)$ & $27.0(16.0-48.0)^{*}$ & 0.48 \\
UBE (repetitions) $_{\text {VO }}$ (meak $\left(\mathrm{mL} / \mathrm{kg} \cdot \mathrm{min}^{-1}\right)$ & $18.0(3.0-40.0)$ & $22.5(4.0-43.0)$ & 0.23 \\
\hline
\end{tabular}

$\mathrm{ES}=\mathrm{Effect}$ size; $\mathrm{BMI}=$ Body mass index; $\mathrm{WC}=$ Waist circumference; $\mathrm{AE}=$ Abdominal endurance; $\mathrm{UBE}=$ Upper body endurance. ${ }^{\star}=$ Significant difference between pre and post yoga program $(p<0.01)$. 
with previous studies that observed a decrease in WC after a 6-day yoga and diet program (Telles, Naveen, Balkrishna, \& Kumar, 2010), in fat percentage during the practice of hatha yoga (Bhutkar, Bhutkar, Taware, \& Surdi, 2011; Ramos-Jiménez. Hernandéz-Torres, \& Wall-Mendrano, 2011), and an increase in flexibility with 6 or 8 weeks of yoga classes (Tran, Holly, Lashbrook, \& Amsterdam, 2001; Cowen \& Adams, 2005; Cowen, 2010; Hewett, Ransdell, Gao et al., 2011; Amin \& Goodman, 2014). Nevertheless, WC reduction after yoga program is also controversial in different population (Hedge, Adhikari, Kotian et al., 2011). Lastly, both body mass and cardiorespiratory test did not show statistical difference after the intervention program.

WC is an important measure of central obesity, and there is a strong association between WC, cardiovascular and metabolic risk factors (Zimmet, Magliano, Matsuzawa et al., 2005). In that sense, Telles, Naveen, Balkrishna, and Kumar (2010) evaluated the impact of 6-day yoga program on the anthropometric measures of obese persons with controlled diet. Although the program applied by that researchers was shorter than the present study, it was demonstrated an important reduction about approximately $2 \mathrm{~cm}$ in $\mathrm{WC}$ after the protocol. The reduction showed in the present study was about approximately $3 \mathrm{~cm}$ after the protocol, moreover the intervention period was longer. Noteworthy, the participants in the present study were young and healthy adults without diet control. Perhaps, the sample baseline might explain these similar results, even with different duration in the intervention program. In contrast, after evaluate 60 subjects during 12-week yoga program in type 2 diabetes mellitus of both gender, Hedge, Adhikari, Kotian et al. (2011) did not find reduction on WC. Beyond the lack of available studies analyzing this variable, it is reasonable to assume that WC response to a yoga program might depend on the sample characteristics and duration of the intervention. Curiously, Hedge, Adhikari, Kotian et al. (2011) and Bhutkar, Bhutkar, Taware, and Surdi (2011) found reduction in BMI, suggesting yoga intervention as a positive tool to improve BMI pattern. It is worthy to mention that even small reduction on BMI can lead to important health improvements as a reduction on the risk for metabolic syndrome (Mileski, Leitão, Lofrano-Porto, \& Grossi Porto, 2015). However it should be highlighted that almost all volunteers of our sample were in a normal range of BMI, what may explain the lack of effect on that variable.

The body fat percentage represents another important measure of obesity that was significantly improved after the intervention. Recently, Indian researchers followed young adults (49 males and 30 females), 6 times per week, during 24-week yoga program (Bhutkar, Bhutkar, Taware, \& Surdi, 2011). After the protocol, females but not males reduced significantly the body fat percentage in approximately $-2 \%$. The authors attributed these findings to the different diets between groups, although the feeding had not been controlled during the intervention program. Nevertheless, the sample of present study was almost composed by females, although there were no differences between gender groups when it was analyzed separately. Ramos-Jiménez, Hernandéz-Torres, and Wall- 
Medrano (2011) also investigated the effects of a yoga program during 11 weeks on body composition of Mexican adult women. The protocol consisted in exposing 16 experienced yoga practitioners to 75 minutes of yoga class, 5 times per week. After the protocol and corroborating our findings, the researchers observed body fat reduction even in trained practitioners (more than 3 years of experience). Besides of the good yoga health results observed in ours and other studies, its effects on body composition need investigations in bigger samples and for longer periods of time.

Ramos-Jiménez, Hernandéz-Torres, and Wall-Medrano (2011) also observed $\mathrm{VO}_{\text {2peak }}$ improvement after their yoga intervention, in contrast to our findings. In addition, there are other studies that have shown benefits of yoga practice for cardiopulmonary endurance in healthy people (Birkel \& Edgren, 2000; Ray, Mukhopadhyaya, Purkayastha et al., 2001; Tran, Holly, Lashbrook, \& Amsterdam, 2001). The rationale hypothesis to the cardiorespiratory improvement associated with yoga training is an expected physiological adaptation related to an increased oxygen delivery, decreased oxygen consumption and respiration rate, and decreased resting heart rate for a same effort level, resulting in improved exercise capacity (Raub, 2002). Similarly to our findings, Tracy and Hart (2013) have not found changes in $\mathrm{VO}_{2} \max$ in healthy young adults yoga practitioners, after eight weeks of intervention. The intensity of exercise performed in a traditional yoga class is low, as shown by Ray, Pathak, and Tomer (2011), who have found a range from $9.9 \%$ to $26.5 \%$ of $\mathrm{VO}_{2}$ max during a full yoga practice session. The metabolic costs during one hour are similar to walking on a treadmill at 3.2 $\mathrm{kph}$, and may not meet recommendations for levels of physical activity for improving or maintaining health or cardiovascular fitness (Hagins, Moore, \& Rundle, 2007). Perhaps, it could be understood as a result of the slow execution of the postures with adequate rest pause; as a consequence, it may be not enough to promote significant changes in $\mathrm{VO}_{2} \mathrm{max}$, except for the practice of sun salutation, which is a dynamic exercise and may promote conditioning of cardiorespiratory parameters (Sinha \& Sinha, 2014). Another possibility to take into account is the relatively short period of intervention to allow an improvement in cardiorespiratory fitness in young adults.

It was observed a small effect size considering upper body endurance, however and contrarily, other studies have shown significant improvement in push-up (Cowen \& Adams, 2005; Bhutkar, Bhutkar, Taware, \& Surdi, 2011). Bhutkar, Bhutkar, Taware, and Surdi (2011), however, designed their study with the performance of only sun salutation, a slow dynamic exercise. Many of its poses contribute to strength because they require sustained contractions of large muscle groups, which are comparable to resistance training (Campbell, Crim, Young, \& Evans, 1994). The lack of specificity for the protocol used in the present study may explain this result on upper body endurance. Another major finding was that abdominal endurance may have improved as a result of this Hatha Yoga intervention, and it is corroborated by other preview studies (Cowen \& Adams, 2005; Bhutkar, Bhutkar, Taware, \& Surdi, 2011). Perhaps, it has 
happened due to the fact that yoga exercises the trunk region and requires the core musculature. In addition, yoga can influence reducing this local fat. That combination could explain an increase in strength and endurance of abdominal muscles (Bera \& Rajapurkar, 1993).

It is necessary recognize that the present study had some methodological limitations, as a small self-selected sample, the absence of a control group, and the withdrawal of subjects before the post-test. Yet the relative magnitude and consistency of the observed differences between pre and post-test support their validity. In contrast to prior studies, it was used the same sequence of yoga postures during all the intervention period. Intervention with yoga is much recommended, since it is easy to practice, it does not require any tools, and limited space is enough. Future studies could incorporate a control group, and a physically active experimental group. Also, additional suggestions for future research include analyzing dose response relationship for yoga, ideal frequency and duration in postures. Albeit these limitations, the observed improvement in waist circumference, body fat, flexibility and abdominal endurance associated with a 12 -week period of yoga training should be carefully taken into account in a scenario of an obesity and physical inactivity pandemic. As yoga seems to have good adherence as compared to more traditional physical activities programs, its effects on health-related physical fitness should be focused on furthers studies. If our results are confirmed on lager and better-controlled studies, yoga interventions may represent a powerful alternative in physical activity interventions focused on a public health approach.

\section{Conclusion}

Results of the present study indicate that a 12-week yoga intervention was associated with improvement on health-related physical fitness in young healthy subjects. More specifically, the applied yoga program was associated with significant decrease of WC and relative body fat, and increase of hamstring flexibility and abdominal endurance.

\section{Grants or Other Financial Support}

Luiz Guilherme Grossi Porto is a scholarship from the Conselho Nacional de Desenvolvimento Científico e Tecnológico-CNPq-Brazil.

\section{References}

ACSM (2011). Quantity and Quality of Exercise for Developing and Maintaining Cardiorespiratory, Musculoskeletal, and Neuromotor Fitness in Apparently Healthy Adults: Guidance for Prescribing Exercise. Medicine \& Science in Sports \& Exercise, 43, 1334 1359. https://doi.org/10.1249/MSS.0b013e318213fefb

Aljasir, B., Bryson, M., \& Al-Shehri, B. (2008). Yoga Practice for the Management of Type II Diabetes Mellitus in Adults: A Systematic Review. Evidence-Based Complementary and Alternative Medicine, 7, 399-408. https://doi.org/10.1093/ecam/nen027

Allen, K., \& Morey, M. C. (2010). Physical Activity and Adherence. In H. B. Bosworth (Ed.), Improving Patient Treatment Adherence: A Clinician's Guide. New York: Sprin- 
ger. https://doi.org/10.1007/978-1-4419-5866-2_2

Amin, D. J., \& Goodman, M. (2014). The Effects of Selected Asanas in Iyengar Yoga on Flexibility: Pilot Study. Journal of Bodywork and Movement Therapies, 18, 399-404. https://doi.org/10.1016/j.jbmt.2013.11.008

Bankar, M. A., Chaudhari, S. K., \& Chaudhari, K. D. (2013). Impact of Long Term Yoga Practice on Sleep Quality and Quality of Life in the Elderly. Journal of Ayurveda and Integrative Medicine, 4, 28-32. https://doi.org/10.4103/0975-9476.109548

Bera, T. K., \& Rajapurkar, M. V. (1993). Body Composition, Cardiovascular Endurance and Anaerobic Power of Yogic Practitioner. Indian Journal of Physiology and Pharmacology, 37, 225-228.

Bhutkar, M. V., Bhutkar, P. M., Taware, G. B., \& Surdi, A. D. (2011). How Effective Is Sun Salutation in Improving Muscle Strength, General Body Endurance and Body Composition? Asian Journal of Sports Medicine, 2, 259-266. https://doi.org/10.5812/asjsm.34742

Birdee, G. S., Legedza, A. T., Saper, R. B., Bertisch, S. M., Eisenberg, D. M., \& Phillips, R. S. (2008). Characteristics of Yoga Users: Results of a National Survey. Journal of General Internal Medicine, 23, 1653-1658. https://doi.org/10.1007/s11606-008-0735-5

Birkel, D. A., \& Edgren, L. (2000). Hatha Yoga: Improved Vital Capacity of College Students. Alternative Therapies in Health and Medicine, 6, 55-63.

Buffart, L. M., Uffelen, J. G. Z., Riphagen, I. I., Brug, J., Mechelen, W., Brown, W. J., \& Chinapaw, M. J. M. (2012). Physical and Psychosocial Benefits of Yoga in Cancer Patients and Survivors, a Systematic Review and Meta-Analysis of Randomized Controlled Trials. BMC Cancer, 12, 559-580. https://doi.org/10.1186/1471-2407-12-559

Campbell, W. W., Crim, M. C., Young, V. R., \& Evans, W. J. (1994). Increased Energy Requirements and Changes in Body Composition with Resistance Training in Older Adults. The American Journal of Clinical Nutrition, 60, 167-175.

Caspersen, C. J., Powell, K. E., \& Christenson, G. M. (1985). Physical Activity, Exercise, and Physical Fitness: Definitions and Distinctions for Health-Related Research. Public Health Reports, 100, 126-131.

Cooper, K. H. (1968). A Means of Assessing Maximal Oxygen Intake: Correlation between Field and Treadmill Testing. JAMA, 203, 201-204. https://doi.org/10.1001/jama.1968.03140030033008

Cowen, V. S. (2010). Functional Fitness Improvements after a Worksite-Based Yoga Initiative. Journal of Bodywork and Movement Therapies, 14, 50-54. https://doi.org/10.1016/j.jbmt.2009.02.006

Cowen, V. S., \& Adams, T. B. (2005). Physical and Perceptual Benefits of Yoga Asana Practice: Results of a Pilot Study. Journal of Bodywork and Movement Therapies, 9, 211-219. https://doi.org/10.1016/j.jbmt.2004.08.001

Ding, D., \& Stamatakis, E. (2014). Yoga Practice in England 1997-2008: Prevalence, Temporal Trends, and Correlates of Participation. BMC Research Notes, 7, 172-175. https://doi.org/10.1186/1756-0500-7-172

Erikssen, G. (2001). Physical Fitness and Changes in Mortality: The Survival of the Fittest. Sports Medicine, 31, 571-576. https://doi.org/10.2165/00007256-200131080-00001

Field, T. (2011). Yoga Clinical Research Review. Complementary Therapies in Clinical Practice, 17, 1-8. https://doi.org/10.1016/j.ctcp.2010.09.007

Flegal, K. E., Kishiyama, S., Zajdel, D., Haas, M., \& Oken, B. S. (2007). Adherence to Yoga and Exercise Interventions in a 6-Month Clinical Trial. BMC Complementary and Alternative Medicine, 7, 37-43. https://doi.org/10.1186/1472-6882-7-37 
Gadin, K. G., \& Hammarström, A. (2002). Can School-Related Factors Predict Future Health Behaviour among Young Adolescents? Public Health, 116, 22-29. https://doi.org/10.1038/sj.ph.1900807

Hagins, M., Moore, W., \& Rundle, A. (2007). Does Practicing Hatha Yoga Satisfy Recommendations for Intensity of Physical Activity Which Improves and Maintains Health and Cardiovascular Fitness? BMC Complementary and Alternative Medicine, 7, 40-48. https://doi.org/10.1186/1472-6882-7-40

Hallal, P. C., Andersen, L. B., Bull, F. C., Guthold, R., Haskell, W., Ekelund, U. et al. (2012). Global Physical Activity Levels: Surveillance Progress, Pitfalls, and Prospects. The Lancet, 380, 247-257. https://doi.org/10.1016/S0140-6736(12)60646-1

Hedge, S. V., Adhikari, P., Kotian, S., Pinto, V. J., S'Souza, S., \& D’Souza, V. (2011). Effect of 3-Month Yoga on Oxidative Stress in Type 2 Diabetes with or without Complications: A Controlled Clinical Trial. Diabetes Care, 34, 2208-2210.

https://doi.org/10.2337/dc10-2430

Hewett, Z. L., Ransdell, L. B., Gao, Y., Petlichkoff, L. M., \& Lucas, S. (2011). An Examination of the Effectiveness of an 8-Week Bikram Yoga Program on Mindfulness, Perceived Stress, and Physical Fitness. Journal of Exercise Science and Fitness, 9, 87-92. https://doi.org/10.1016/S1728-869X(12)60003-3

Hoeger, W. K., \& Hoeger, S. A. (2010). Principles and Labs for Fitness and Wellness. Belmont, CA: Wadsworth.

Kristal, A. R., Littman, A. J., Benitez, D., \& White, E. (2005). Yoga Practice Is Associated with Attenuated Weight Gain in Healthy, Middle Aged Men and Women. Alternative Therapies, 11, 28-33.

Lee, I. M., Shiroma, E. J., Lobelo, F., Puska, P., Blair, S. N., Katzmarzyk, P. T. et al. (2012). Effect of Physical Inactivity on Major Non-Communicable Diseases Worldwide: An Analysis of Burden of Disease and Life Expectancy. The Lancet, 380, 219-229.

https://doi.org/10.1016/S0140-6736(12)61031-9

Matsudo, V. K., Matsudo, S. M., Araújo, T. L., Andrade D. R., Oliveira, L. C., \& Hallal, P. C. (2010). Time Trends in Physical Activity in the State of São Paulo, Brazil: 2002-2008. Medicine Science Sports Exercise, 42, 2231-2236. https://doi.org/10.1249/MSS.0b013e3181e1fe8e

McCall, M., Ward, A., Roberts, N. W., \& Heneghan, C. (2013). Overview of Systematic Reviews: Yoga as a Therapeutic Intervention for Adults with Acute and Chronic Health Conditions. Evidence-Based Complementary and Alternative Medicine, 2013, Article ID: 945895. https://doi.org/10.1155/2013/945895

Mileski, K. S., Leitão, J. L., Lofrano-Porto, A., \& Grossi Porto, L. G. (2015). Health-Related Physical Fitness in Middle-Aged Men with and without Metabolic Syndrome. The Journal of Sports Medicine and Physical Fitness, 55, 223-230.

Posadzki, P., Ernst, E., Terry, R., \& Lee, M. S. (2011). Is Yoga Effective for Pain? A Systematic Review of Randomized Clinical Trials. Complementary Therapies in Medicine, 19, 281-287. https://doi.org/10.1016/j.ctim.2011.07.004

Ramos-Jiménez, A., Hernandéz-Torres, R. P., \& Wall-Medrano, A. (2011). Hatha Yoga Program Determinants on Cardiovascular Health in Physically Active Adult Women. Journal of Yoga and Physical Therapy, 1, 103. https://doi.org/10.4172/2157-7595.1000103

Raub, J. A. (2002). Psychophysiologic Effects of Hatha Yoga on Musculoskeletal and Cardiopulmonary Function: A Literature Review. Journal of Alternative and Complementary Medicine, 8, 797-812. https://doi.org/10.1089/10755530260511810

Ray, U. S., Mukhopadhyaya, S., Purkayastha, S. S., Asnani, V., Tomer, O. S., Prashad, R., 
Thakur, L., \& Selvamurthy, W. (2001). Effect of Yogic Exercises on Physical and Mental Health of Young Fellowship Course Trainees. Indian Journal of Physiology and Pharmacology, 45, 37-53.

Ray, U. S., Pathak, A., \& Tomer, O. S. (2011). Hatha Yoga Practices: Energy Expenditure, Respiratory Changes and Intensity of Exercise. Evidence-Based Complementary and Alternative Medicine, 2011, Article ID: 241294.

https://doi.org/10.1093/ecam/neq046

Rioux, J. G., \& Ritenbaugh, C. (2013). Narrative Review of Yoga Intervention Clinical Trials Including Weight-Related Outcomes. Alternative Therapies in Health and Medicine, 19, 32-46.

Rooney, E. M. (1993). Exercise for Older Patients: Why It's Worth Your Effort. Geriatrics, 48, 68-77.

Seo, D. Y., Lee, S., Figueroa, A., Kim, H. K., Baek, Y. H., Kwak, Y. S., Kim, N. et al. (2012). Yoga Training Improves Metabolic Parameters in Obese Boys. Korean Journal of Physiology and Pharmacology, 16, 175-180. https://doi.org/10.4196/kjpp.2012.16.3.175

Sinha, B., \& Sinha, T. D. (2014). Effect of 11 Months of Yoga Training on Cardiorespiratory Responses during the Actual Practice of Surya Namaskar. International Journal of Yoga, 7, 72-75. https://doi.org/10.4103/0973-6131.123493

Taylor, D. (2014). Physical Activity Is Medicine for Older Adults. Postgraduate Medical Journal, 90, 26-32. https://doi.org/10.1136/postgradmedj-2012-131366

Telles, S., Naveen, V. K., Balkrishna, A., \& Kumar S. (2010). Short Term Health Impact of a Yoga and Diet Change Program on Obesity. Medical Science Monitor, 16, 35-40.

Torres, A., Sarmiento, O. L., Stauber, C., \& Zarama, R. (2013). The Ciclovia and Cicloruta Programs: Promising Interventions to Promote Physical Activity and Social Capital in Bogotá, Colombia. American Journal of Public Health, 103, 23-30. https://doi.org/10.2105/AJPH.2012.301142

Tracy, B. L., \& Hart, C. E. F. (2013). Bikram Yoga Training and Physical Fitness in Healthy Young Adults. Journal of Strength \& Conditioning Research, 27, 822-830. https://doi.org/10.1519/JSC.0b013e31825c340f

Tran, M. D., Holly, R. G., Lashbrook, J., \& Amsterdam, E. A. (2001). Effects of Hatha Yoga Practice on the Health-Related Aspects of Physical Fitness. Preventive Cardiology, 4, 165-170. https://doi.org/10.1111/j.1520-037X.2001.00542.x

Woodyard, C. (2011). Exploring the Therapeutic Effects of Yoga and Its Ability to Increase Quality of Life. International Journal of Yoga, 4, 49-54. https://doi.org/10.4103/0973-6131.85485

Yang, K. (2007). A Review of Yoga Programs for Four Leading Risk Factors of Chronic Diseases. Evidence-Based Complementary and Alternative Medicine, 4, 487-491. https://doi.org/10.1093/ecam/nem154

Zimmet, P., Magliano, D., Matsuzawa, Y., Alberti, G., \& Shaw, J. (2005). The Metabolic Syndrome: A Global Public Health Problem and a New Definition. Journal of Atherosclerosis and Thrombosis, 12, 295-300. https://doi.org/10.5551/jat.12.295 
Submit or recommend next manuscript to SCIRP and we will provide best service for you:

Accepting pre-submission inquiries through Email, Facebook, LinkedIn, Twitter, etc. A wide selection of journals (inclusive of 9 subjects, more than 200 journals)

Providing 24-hour high-quality service

User-friendly online submission system

Fair and swift peer-review system

Efficient typesetting and proofreading procedure

Display of the result of downloads and visits, as well as the number of cited articles Maximum dissemination of your research work

Submit your manuscript at: http://papersubmission.scirp.org/

Or contact ape@scirp.org 